\title{
Effect of membrane exposure on guided bone regeneration: $A$ systematic review and meta-analysis
}

\author{
Jeffrey Garcia ${ }^{1}$ | Austin Dodge ${ }^{1}$ | Paul Luepke ${ }^{1}$ | Hom-Lay Wang ${ }^{2}$ (D) | Yvonne \\ Kapila $^{3}$ | Guo-Hao Lin ${ }^{1,3}$ (C)
}

${ }^{1}$ Department of Surgical Sciences, Marquette University School of Dentistry, Milwaukee, WI, USA

${ }^{2}$ Graduate Periodontics, Department of Periodontics \& Oral Medicine, University of Michigan School of Dentistry, Ann Arbor, MI, USA

${ }^{3}$ Department of Orofacial Sciences, School of Dentistry, University of California San Francisco, San Francisco, CA, USA

\section{Correspondence}

Guo-Hao Lin, DDS, MS, Health Sciences Assistant Clinical Professor, Department of Orofacial Sciences, University of California, San Francisco, CA, USA.

Email: ghlin@umich.edu; guo-hao.lin@ucsf.edu

\begin{abstract}
Aims: This review aimed at investigating the effect of membrane exposure on guided bone regeneration (GBR) outcomes at peri-implant sites and edentulous ridges.

Material and Methods: Electronic and manual literature searches were conducted by two independent reviewers using four databases, including MEDLINE, EMBASE, Web of Science, and Cochrane Central Register of Controlled Trials, for articles up to February 2017. Articles were included if they were human clinical trials or case series reporting outcomes of GBR procedures with and without membrane exposure. A random-effects meta-analysis was conducted, and the weighted mean difference (WMD) between the two groups and 95\% confidence interval $(\mathrm{Cl})$ were reported.

Results: Overall, eight articles were included in the quantitative analysis. The WMD of the horizontal bone gain at edentulous ridges was $-76.24 \%(95 \% \mathrm{Cl}=-137.52 \%$ to $-14.97 \%, p=.01$ ) between sites with membrane exposure and without exposure. In addition, the WMD of the dehiscence reduction at peri-implant sites was $-27.27 \%$ (95\% Cl of $-45.87 \%$ to $-8.68 \%, p=.004)$. Both analyses showed significantly favorable outcomes at the sites without membrane exposure.

Conclusion: Based on the findings of this study, membrane exposure after GBR procedures has a significant detrimental influence on the outcome of bone augmentation. For the edentulous ridges, the sites without membrane exposure achieved $74 \%$ more horizontal bone gain than the sites with exposure. For peri-implant dehiscence defects, the sites without membrane exposure had $27 \%$ more defect reduction than the sites with exposure.
\end{abstract}

\section{KEYWORDS}

alveolar ridge augmentation, bone regeneration, evidence-based dentistry, meta-analysis, review, surgical wound dehiscence

\section{1 | INTRODUCTION}

Alveolar ridge dimensions provide the foundation for primary implant stability and long-term implant success. Bone deficiencies present an immediate concern for clinicians and need to be addressed early in the treatment planning process. The etiology of ridge deficiencies can be either anatomic or pathologic in nature, as described by Buser, Martin, and Belser (2004). In an attempt to standardize defect parameters, several authors have developed classification systems (Allen, Gainza, Farthing, \& Newbold, 1985; Seibert, 1983; Wang \& Al-Shammari, 2002). Each author described three different types of ridge deficiencies based on the progression of the ridge resorption following edentulism: horizontal, vertical, and a combination of the two dimensions. When evaluating an edentulous 
site for future implant placement, ideal treatment outcomes demand sufficient horizontal and vertical ridge dimensions. To prevent further bone remodeling after implant placement, Spray, Black, Morris, and Ochi (2000) proposed a need for $1.8 \mathrm{~mm}$ of bone thickness at the buccal aspect of the implant and $0.5 \mathrm{~mm}$ at the palatal aspect to ensure long-term success. In addition, Tarnow, Cho, and Wallace (2000) also recommended a need for at least $1.5 \mathrm{~mm}$ of distance between an implant and the adjacent root, and at least $3 \mathrm{~mm}$ of distance between two adjacent implants, to accommodate ideal interproximal bone levels as well as for papilla support.

Several techniques have been proposed and widely used to augment deficient ridges. Guided bone regeneration (GBR) is one of the most utilized techniques, and it consists of using grafting materials in combination with a barrier, either a nonresorbable membrane (Buser, Bragger, Lang, \& Nyman, 1990) or an absorbable membrane (Mellonig \& Nevins, 1995). Other techniques include the use of a bone block graft (Misch, 1997) or a ridge-split technique (Simion, Baldoni, \& Zaffe, 1992). Additionally, the use of distraction osteogenesis to augment the edentulous ridge has also been proposed (Chiapasco, Romeo, \& Vogel, 2001; Chin, 1999). Recently, the "sandwich" technique has been described to regenerate horizontal and vertical bone defects at peri-implant sites (Wang, Misch, \& Neiva, 2004). All these techniques are effective; however, complications can occur during the healing phase of treatment, creating undesired outcomes.

Successful GBR procedures are dependent on four fundamental principles that must ensue during the surgery and throughout healing. These principles consist of primary closure, angiogenesis, space maintenance, and stability of the wound, the so-called PASS principle (Wang \& Boyapati, 2006). Complications with any of these principles can result in premature membrane exposure that potentially compromises the regenerative process. To minimize the risk of complications, clinicians should assess the amount of keratinized mucosa, tissue biotype, vestibular depth, flap flexibility, bone defect type and size, and type of membrane used (Chao, Chang, Fu, Wang, \& Chan, 2015). Each one of these factors has been identified as a contributing factor in membrane exposure.

As there is a need to understand the influence of membrane exposure on GBR outcomes, the goal of this study was to compare the amount of bone gain after GBR procedures between sites with and without membrane exposure. The primary outcome was the percentage of horizontal bone gain at edentulous ridges. The secondary outcome was the percentage of peri-implant bone dehiscence reduction at peri-implant sites.

\section{2 | MATERIAL AND METHODS}

\section{1 | Focused question}

What is the effect of membrane exposure on bone augmentation outcomes after horizontal ridge augmentation at edentulous sites after GBR procedures or at peri-implant sites immediately after implant placement?

\section{2 | PICO question (problem, intervention, comparison, outcome)}

P: Maxillary or mandibular partially edentulous healthy subjects who were to receive or had received dental implants to restore oral function

I: GBR for horizontal ridge augmentation to augment bone width for future implant placement or to restore peri-implant dehiscence defects immediately after implant placement

C: GBR outcomes between sites with and without membrane exposure O:

- Primary outcome: percentage of horizontal bone gain at sites with and without membrane exposure at edentulous ridges

- Secondary outcome: percentage of peri-implant bone dehiscence reduction at sites with and without membrane exposure at peri-implant sites

\section{3 | Information sources}

Electronic and manual literature searches were conducted by two independent reviewers (JG and $A D$ ) in four databases, including MEDLINE, EMBASE, Web of Science, and Cochrane Central Register of Controlled Trials, for articles up to February 2017. Two reviewers independently extracted the data from studies (JG and AD). Publications that did not meet the inclusion criteria were excluded. In case of disagreements, consensus was reached by discussion with a third reviewer (GL).

\subsection{Screening process and data extraction}

For the PubMed library, combinations of controlled terms ([mh] represented $\mathrm{MeSH}$ terms) and keywords ([tiab] represented title/abstract search and [all] represented full-text search) were used whenever possible. As such, the key terms used were as follows:

("alveolar ridge augmentation"[all] OR "ridge augmentation"[all] OR "guided bone regeneration"[all])

AND

("dental implants"[mh] OR "absorbable implants"[mh] OR implant [tiab] OR implants [tiab]) AND

("complication"[all] OR "complications"[all] OR "exposure"[all])

For the other databases, the key terms used for the search included GBR, alveolar ridge augmentation, dental implants, complication, and exposure.

The screening in such databases was limited to "clinical studies" AND "humans" in all of the screening strategies. In addition, an electronic screening of the grey literature at the New York Academy of Medicine Grey Literature Report (http://greylit.org) and Google Scholar was conducted as recommended by high standards for systematic reviews, that is Assessment of Multiple Systematic Reviews (AMSTAR) guidelines (Shea et al., 2007).

Additionally, a manual search of periodontics-related journals, including Clinical Implant Dentistry and Related Research, The International Journal of Oral \& Maxillofacial Implants, Clinical Oral Implants Research, 
Implant Dentistry, European Journal of Oral Implantology, Journal of Implantology, International Journal of Oral and Maxillofacial Surgery, Journal of Oral and Maxillofacial Surgery, Journal of Dental Research, Journal of Clinical Periodontology, Journal of Periodontology, and The International Journal of Periodontics \& Restorative Dentistry, from January 2016 up to March 2017, was performed to ensure a thorough screening process. This systematic review was registered at the PROSPERO website (registration number: CRD42017059598).

\section{5 | Inclusion criteria}

Studies were included for the review if the following inclusion criteria were fulfilled: clinical human prospective or retrospective study, number of study sites of $\geq 10$, GBR procedure for ridge augmentations at partially edentulous ridges or peri-implant defects immediately after implant placement. Studies with nonparticulate bone grafting materials (i.e., block grafts), no information regarding complications, augmentation procedures other than GBR (distraction osteogenesis, sinus lifts, etc.), vertical ridge augmentation, and treatment of periimplantitis were excluded from this study. Article titles and abstracts were screened first for inclusion eligibility.

\section{6 | Data extraction}

Data recorded for each study included the study design, number of participants, bone grafting materials used, membrane type selected, initial bone width, final bone width, timing of complication onset, and duration of follow-up after the augmentation surgery.

\section{7 | Data analyses}

The primary outcome was the percentage of horizontal bone gain at edentulous sites in groups with and without membrane exposure. The secondary outcome was the percentage of dehiscence defect reduction at peri-implant sites in groups with and without membrane exposure. The pooled weighted mean difference (WMD) of the percentage of horizontal bone changes at edentulous sites, and the percentage defect depth reduction at peri-implant sites was estimated using a computer program (RevMan version 5.0, The Nordic Cochrane Centre, The Cochrane Collaboration, Copenhagen, 2008). The contribution of each article was weighed. Random effects meta-analyses were applied to the selected studies to manage the inherent variability of results due to a great variety of study designs employed by different research groups. Forest plots were generated to graphically represent the difference in primary and secondary outcomes for all included studies using augmented sites as the analysis unit. $\mathrm{A} p$ value $=.05$ was used as the level of significance. Heterogeneity was assessed with a chi-square test and $l^{2}$ test. Heterogeneity values range between $0 \%$ and $100 \%$, where lower values represent less heterogeneity. In addition, funnel plots were used to assess the presence of publication bias. The data presented in this systematic review adhere to the PRISMA (Preferred Reporting Items for Systematic Review and Meta-Analyses) statement (Liberati et al., 2009).

\section{8 | Risk of bias assessment}

The criteria used to assess the quality of the selected randomized control trials (RCTs) were modified from the checklist of the Cochrane Center (Higgins \& Green, 2011). For non-RCTs, the Methodological Index for Non-Randomized Studies (MINORS) was used to rank the risk of bias of the included studies (Slim et al., 2003). The degree of bias was categorized as follows: low risk if all the criteria were met, moderate risk when only one criterion was missing, and high risk if two or more criteria were missing. Two reviewers (JG and $\mathrm{GL}$ ) assessed all the included articles independently.

\section{3 | RESULTS}

\section{1 | Data extraction}

A total of 837 records were identified through the electronic and manual search process after exclusion of duplicates (Figure 1). Of these, 795 were screened based on the title and/or abstract content, and 42 were selected for full-text assessment and inclusion in the qualitative evaluation. Finally, 34 studies (Antoun, Sitbon, Martinez, \& Missika, 2001; von Arx \& Buser, 2006; von Arx \& Kurt, 1999; Assenza et al., 2001; Bahat \& Fontanessi, 2001; Buser, Dula, Hirt, \& Schenk, 1996; Chiapasco, Autelitano, Rabbiosi, \& Zaniboni, 2013; Cordaro, Amade, \& Cordaro, 2002; Cordaro, Torsello, Morcavallo, \& di Torresanto, 2011; Friedmann, Strietzel, Maretzki, Pitaru, \& Bernimoulin, 2002; Her, Kang, \& Fien, 2012; Kfir, Kfir, \& Kaluski, 2007; Lee, Kim, Yi, \& Choi, 2013; Lindfors, Tervonen, Sandor, \& Ylikontiola, 2010; Lizio, Corinaldesi, \& Marchetti, 2014; Lorenzoni, Pertl, Polansky, Jakse, \& Wegscheider, 2002; Louis, Gutta, Said-Al-Naief, \& Bartolucci, 2008; Lyford, Mills, Knapp, Scheyer, \& Mellonig, 2003; Mattout \& Mattout, 2000; Meloni et al., 2017; Merli et al., 2015; Miyamoto, Funaki, Yamauchi, Kodama, \& Takahashi, 2012; Park, Brooks, Oh, \& Wang, 2009; Parodi, Carusi, Santarelli, \& Nanni, 1998; Proussaefs \& Lozada, 2003, 2006;

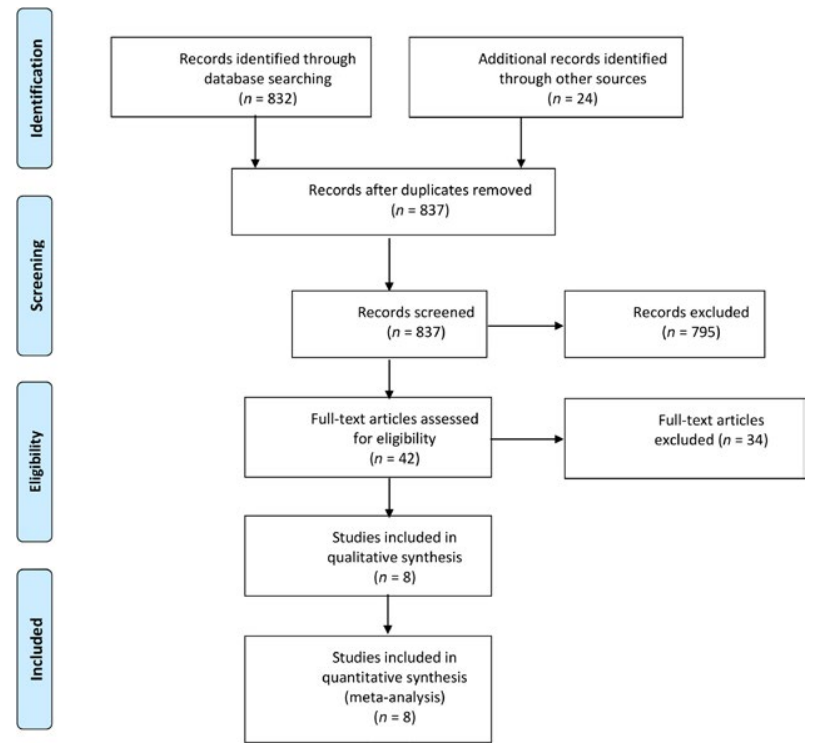

FIGURE 1 PRISMA flowchart demonstrates the inclusion studies selection process 
Proussaefs, 2003; Sethi \& Kaus, 2001; Torres et al., 2010; Uehara et al., 2015; Urban, Nagursky \& Lozada, 2011; Urban, Nagursky, Lozada, \& Nagy, 2013; Wessing, Emmerich, \& Bozkurt, 2016; Zitzmann, Naef, \& Scharer, 1997) were excluded from the quantitative analysis due to lack of data, and eight studies (Annibali, Bignozzi, Sammartino, La Monaca, \& Cristalli, 2012; Buser et al., 1990; Chiapasco, Abati, Romeo, \& Vogel, 1999; Fu, Oh, Benavides, Rudek, \& Wang, 2014; Gher, Quintero, Assad, Monaco, \& Richardson, 1994; Nowzari \& Slots, 1995; Park et al., 2008; Tawil, El-Ghoule, \& Mawla, 2001) that reported on the primary and secondary outcomes were meta-analyzed. The kappa value between the two reviewers was 0.92 for titles and abstract evaluation, and 0.88 for full-text evaluation. The reasons for exclusion of specific studies are noted in Table S1, and the main features of the included studies are summarized in Table 1.

\subsection{Features of the included studies}

\subsection{1 | Study design and patient features}

Three RCTs (Fu et al., 2014; Gher et al., 1994; Park et al., 2008), four prospective case series (PCS; Buser et al., 1990; Chiapasco et al., 1999; Nowzari \& Slots, 1995; Tawil et al., 2001), and one retrospective case series (RCS; Annibali et al., 2012) were included in this study. The age range of the patients in the selected studies was $17-85$ years. All the studies indicated the participants were systemically healthy, and only six studies (Annibali et al., 2012; Chiapasco et al., 1999; Fu et al., 2014; Gher et al., 1994; Nowzari \& Slots, 1995; Park et al., 2008) provided more detailed exclusion criteria: Annibali et al. (2012) and Park et al. (2008) excluded patients who smoked 10 or more cigarettes a day and patients with full-mouth plaque and bleeding scores of $>25 \%$; Chiapasco et al. (1999) excluded heavy smokers, alcohol abusers, patients with poor oral hygiene or active periodontal disease, history of head and neck malignancies, uncontrolled diabetes, severe liver or renal disease, and immune system disorders; Fu et al. (2014) excluded all patients who were smokers, pregnant, or had unstable periodontal diseases, or a history of drug or alcohol abuse; Gher et al. (1994) excluded pregnant patients; and Nowzari and Slots (1995) excluded patients who had antibiotic therapy within 6 months of the study period. Two studies (Annibali et al., 2012; Chiapasco et al., 1999) included patients who were light smokers ( $<10$ cigarettes per day).

The initial bone dimensions were provided by most of the studies, except for three (Fu et al., 2014; Gher et al., 1994; Park et al., 2008). The follow-up period after the GBR procedures, ranging from 4 to 14 months, was reported by all the included studies. Among the included articles, all studies reported no conflict of interest, except one study (Park et al., 2008), which reported a conflict of interest with two private corporations.

\subsection{2 | Types of membranes used}

All the included studies used barrier membranes for GBR procedures. Only two studies (Buser et al., 1990; Chiapasco et al., 1999) that measured horizontal bone gain at edentulous ridges utilized expanded polytetrafluoroethylene (e-PTFE) membranes. Of the studies analyzing the reduction in bone dehiscences at peri-implant sites, two studies (Gher et al., 1994; Nowzari \& Slots, 1995) exclusively used e-PTFE membranes. One study (Annibali et al., 2012) used either titaniumreinforced e-PTFE or absorbable membranes; however, all membrane exposures occurred at sites with e-PTFE barriers. The other three studies (Fu et al., 2014; Park et al., 2008; Tawil et al., 2001) only used absorbable membranes. Tawil et al. (2001) used an absorbable collagen membrane, Park et al. (2008) used either an acellular dermal matrix (ADM) or a collagen membrane, and Fu et al. (2014) introduced a bovine pericardium membrane for GBR procedures.

\subsection{3 | Types of bone grafting materials used}

All included studies, except for one (Nowzari \& Slots, 1995), reported the grafting materials used for the GBR procedures. For studies that measured horizontal bone gain at edentulous sites, one (Chiapasco et al., 1999) used autogenous bone grafts and the other (Buser et al., 1990) did not use bone grafting material at all. In terms of studies that analyzed dehiscence reduction at peri-implant sites, one study (Tawil et al., 2001) solely used autogenous cortical bone, another study (Gher et al., 1994) used demineralized freeze-dried bone allograft (DFDBA), two other studies (Fu et al., 2014; Park et al., 2008) used mineralized allograft, and the other one study (Annibali et al., 2012) used a combination of autogenous bone chips and DFDBA or bovine xenograft.

\section{3 | Incidence of membrane exposure}

For all studies, the exposure of the membrane was clinically detected during the follow-up period. The exposure resulted in surgical removal of the membrane in all but four studies (Fu et al., 2014; Gher et al., 1994; Park et al., 2008; Tawil et al., 2001). Gher et al. (1994) attributed the exposure, which occurred during the first 2 weeks postoperatively, to inappropriate trimming of the membrane, and used $0.12 \%$ chlorhexidine rinses for the duration of the healing time. Two weeks postoperatively, Tawil et al. (2001) resutured the flaps to gain closure and did so without removing the membrane. Park et al. (2008) had five sites with collagen membrane exposure and two sites with ADM exposure. Fu et al. (2014) had three patients with partial cover screw exposure at 2 weeks, yet the surgical site closed completely at the 1-month re-evaluation. The remaining studies reported membrane exposures from 8 to 10 days to 36 weeks postoperatively. Of these, the adverse events of the exposure were reported in two studies: Buser et al. (1990) reported unusual edema, and Nowzari and Slots (1995) reported inflammation, suppuration, and pain. The other two studies (Annibali et al., 2012; Chiapasco et al., 1999) did not comment on the events of the membrane exposure.

\subsection{Meta-analysis of the primary outcome \& secondary outcome}

As the size of the initial bone defect impacts the amount of defect reduction, only studies reporting the percentage of bone defect reduction were pooled for comparable comparisons. 


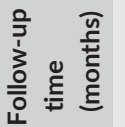

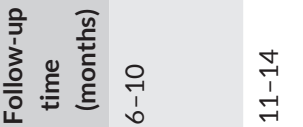

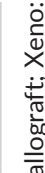

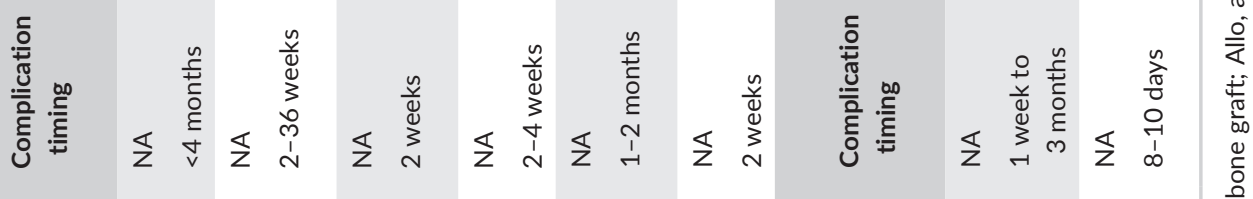

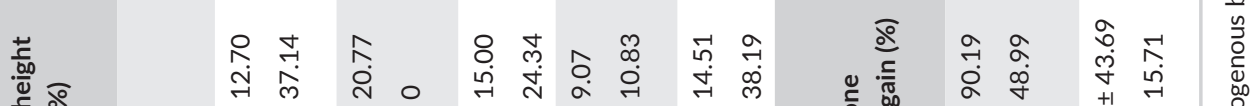

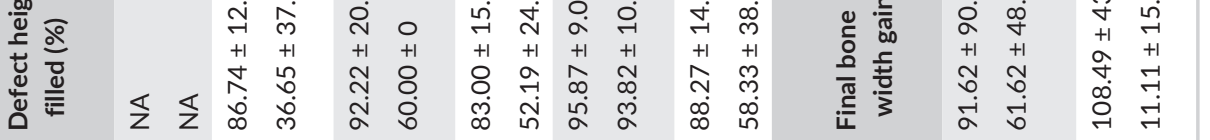

喜

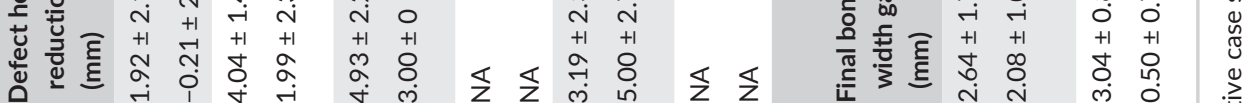

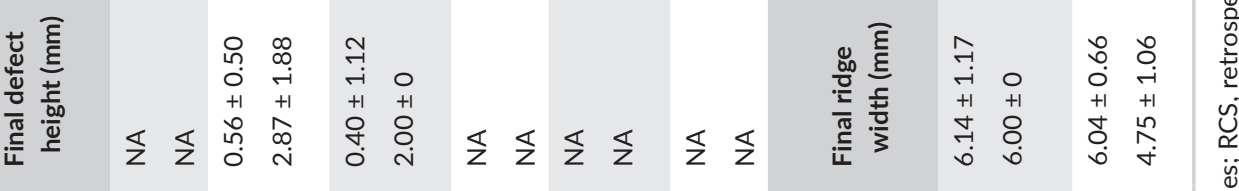

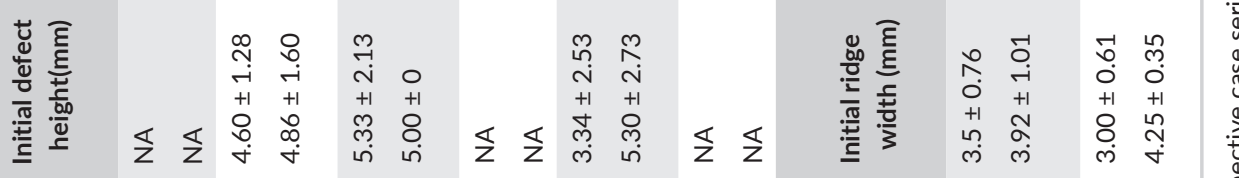

苋

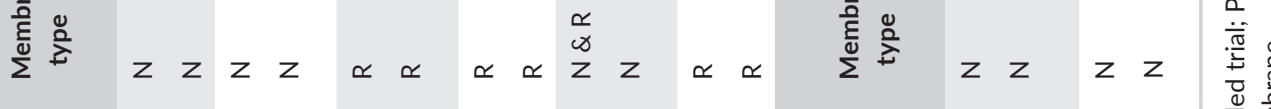

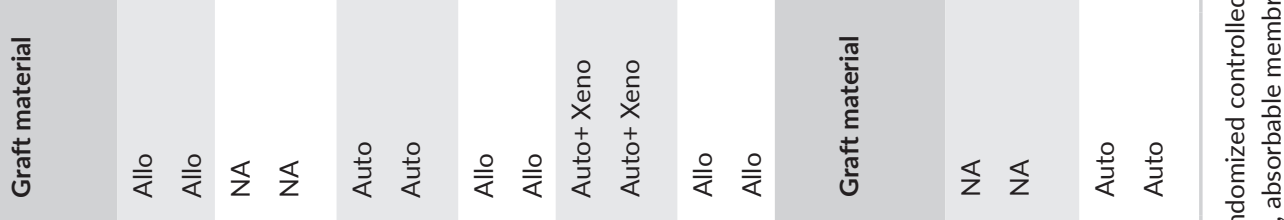

品

竞

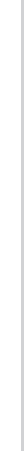

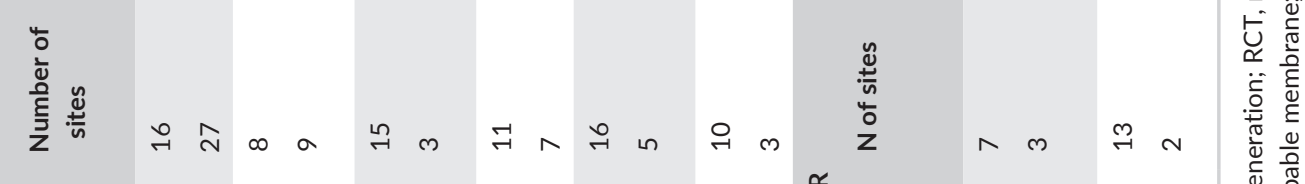

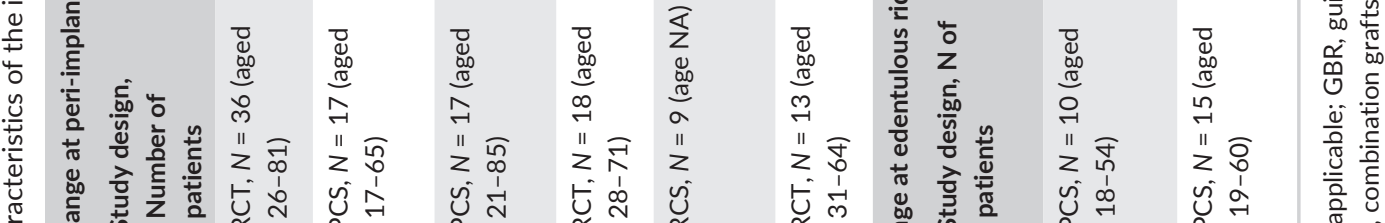

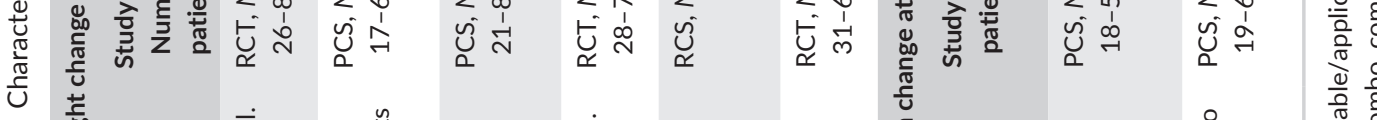

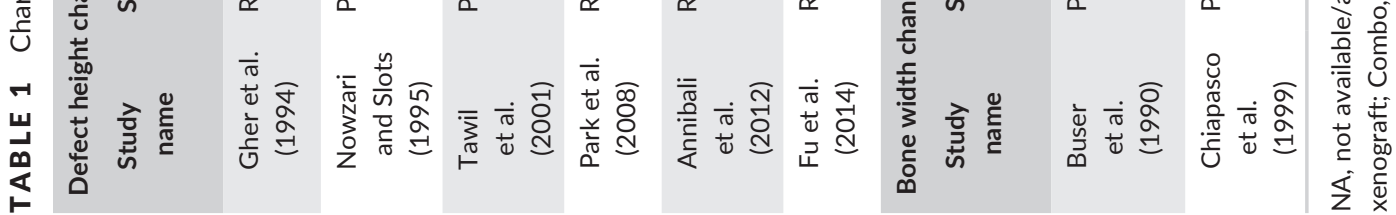




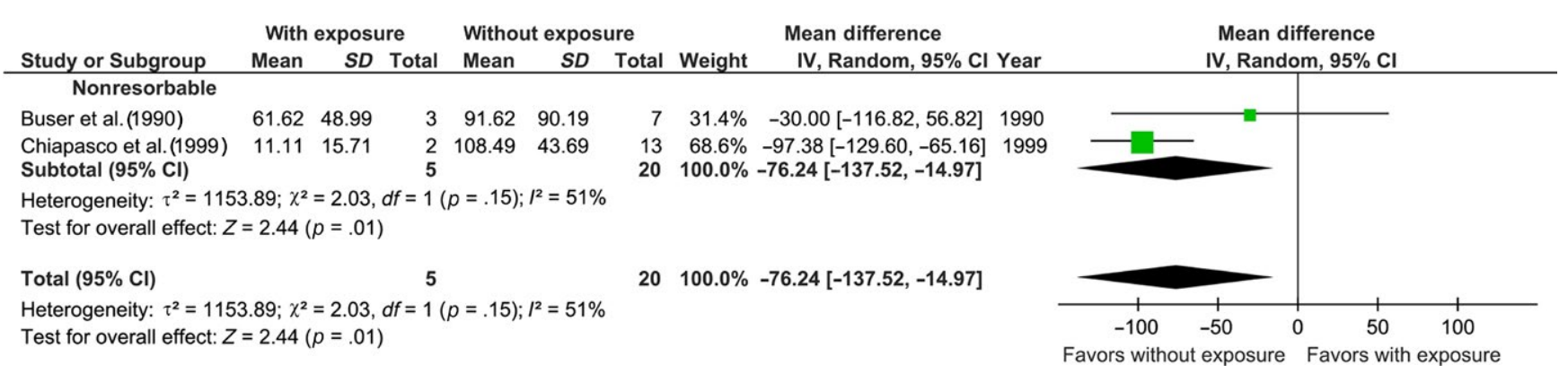

FIGURE 2 Forest plots representing the outcomes of the percentage of horizontal bone width gain after GBR at edentulous ridges between groups with and without membrane exposure. Meta-analysis showed a statistically significant difference (WMD $=-76.24 \%$, with a 95\% Cl of $-137.52 \%$ to $-14.97 \%, p=.01$ ), favoring the group without membrane exposure

Two studies (Buser et al., 1990; Chiapasco et al., 1999) reported outcomes on the percentage of horizontal bone gain after GBR at edentulous sites with and without membrane exposure. Meta-analysis showed a statistically significant difference (WMD $=-76.24 \%$, with a $95 \% \mathrm{Cl}$ of $-137.52 \%$ to $-14.97 \%, p=.01$, Figure 2 ) between the two groups, favoring the group without membrane exposure. A moderate level of heterogeneity was seen ( $p$ value for chi-square test $=.15$ and $I^{2}$ test $\left.=51 \%\right)$ among the pooled studies.

Five studies (Annibali et al., 2012; Fu et al., 2014; Nowzari \& Slots, 1995; Park et al., 2008; Tawil et al., 2001) reported outcomes on the percentage of bone dehiscence reduction after GBR at peri-implant sites. Meta-analysis showed a statistically significant difference $(\mathrm{WMD}=-27.27 \%$, with a $95 \% \mathrm{Cl}$ of $-45.87 \%$ to $-8.68 \%, p=.004$, Figure 3) between the two groups, favoring the group without membrane exposure. A high level of heterogeneity was seen ( $p$ value for chi-square test $=.0001$ and $I^{2}$ test $=83 \%$ ) among the pooled studies. Subgroup analysis based on the type of membranes used was also conducted. For the nonresorbable membrane subgroup, two studies (Annibali et al., 2012; Nowzari \& Slots, 1995) were pooled and meta-analysis showed no statistically significant difference $(\mathrm{WMD}=-24.56 \%$, with a $95 \% \mathrm{Cl}$ of $-71.55 \%$ to $22.42 \%, p=.31$ ). However, this subgroup analysis also revealed a high level of heterogeneity between the two studies ( $p$ value for chi-square test $<.0007$ and $I^{2}$ test $=91 \%$ ). For the absorbable membrane subgroup, three studies (Fu et al., 2014; Park et al., 2008; Tawil et al., 2001) were included, and these revealed a statistically significant difference (WMD $=-31.83 \%$, with a $95 \% \mathrm{Cl}$ of $-40.95 \%$ to $-22.72 \%, p<.0001$ ), favoring the group without membrane exposure. This subgroup analysis revealed a low level of heterogeneity among the pooled studies ( $p$ value for chi-square test $=.99$ and $l^{2}$ test $=0 \%$ ). Funnel plots for the analysis of edentulous ridges and peri-implant sites were reported as Figures S1 and S2.

\section{5 | Risk of bias assessment}

The results of the risk of bias assessment for included case series are summarized in Table 2 (RCT) and Table 3 (non-RCTs). One RCT (Gher et al., 1994) and one case series (Annibali et al., 2012) were considered to have a high risk of bias. Another RCT (Fu et al., 2014) and four case series (Buser et al., 1990; Chiapasco et al., 1999; Nowzari \& Slots, 1995; Tawil et al., 2001) were considered to have a moderate risk of bias. One other RCT was considered to have a low risk of bias (Park et al., 2008). The kappa value of the interexaminer agreement for risk of bias assessment was 1.00

\section{4 | DISCUSSION}

In 2001, Machtei (2001) investigated the effect of early membrane exposure on guided tissue and bone regeneration. The results of the study showed a difference of six times greater bone gain if the healing period did not have early membrane exposure. One of the main drawbacks of that investigation was that only two papers were included in the assessment. Furthermore, the results of the study were reported in millimeters instead of percentages of bone gain, potentially introducing issues with the validity of the comparisons. Without identifying the dimensions of the initial defect size, the amount of potential bone gain measured between the groups could yield incomparable datasets. For example, one of the included studies (Annibali et al., 2012) reported an average of $5.00 \mathrm{~mm}$ of bone gain for sites with membrane exposure, which is greater than the $3.19 \mathrm{~mm}$ gained at sites without membrane exposure. While this seems to suggest almost $2 \mathrm{~mm}$ of difference between the groups, when analyzed by the percentage of defect reduction, the two groups showed comparable percentages of defect reduction, $90.82 \%$ for sites without membrane exposure, and $87.50 \%$ for sites with exposure, respectively. In order to compare the outcomes more meaningfully and to avoid potentially biased analyses, we used the percentage of horizontal bone gain/dehiscence reduction instead of linear measurements as our study outcomes.

The objective of using a barrier membrane is to prevent the ingrowth of soft tissue, while providing space for the graft and allowing oxygen and nutrients to enter the grafted site (Rakhmatia, Ayukawa, Furuhashi, \& Koyano, 2013). Prior to our study, no meta-analysis has been performed to compare the resulting bone gain of edentulous sites under exposed and nonexposed GBR membranes. We found that the sites without membrane exposure achieved $74 \%$ more horizontal bone gain than those with exposure. However, it is worth mentioning that the two articles (Annibali et al., 2012; Nowzari \& Slots, 1995) exclusively used e-PTFE barriers instead of dense PTFE (d-PTFE) barriers. The difference in bacterial resistance between ePTFE and d-PTFE (d-PTFE being more resistant) could be significant. 


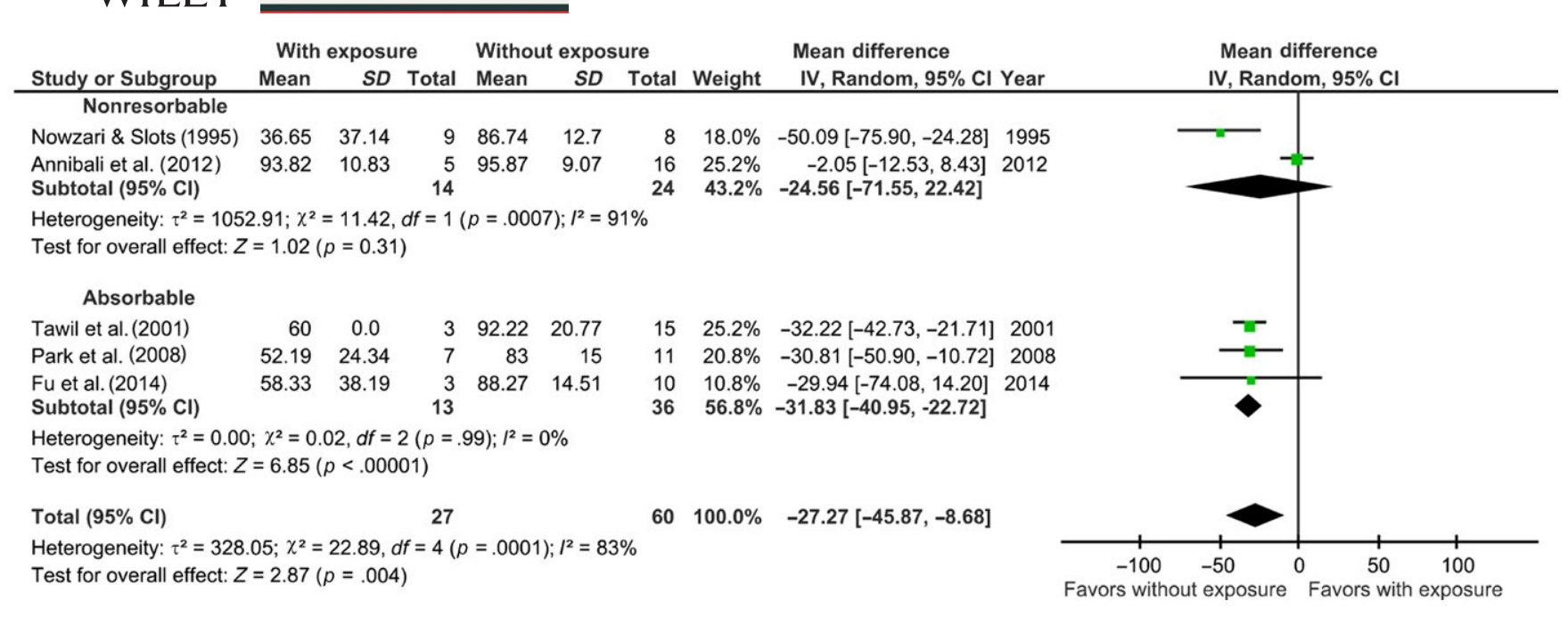

FIGURE 3 Forest plots representing the outcomes of the percentage of peri-implant bone dehiscence reduction after GBR between groups with and without membrane exposure. Meta-analysis showed a statistically significant difference (WMD $=-27.27 \%$, with a $95 \% \mathrm{Cl}$ of $-45.87 \%$ to $-8.68 \%, p=.004$ ), favoring the group without membrane exposure

Studies have shown that intentionally exposed d-PTFE membranes for socket preservation procedures (Greenstein \& Carpentieri, 2015) as well as GBR procedures (Hoffmann et al., 2008; Waasdorp \& Feldman, 2013) did not exhibit significantly compromised regeneration outcomes. As the pore sizes of d-PTFE (less than $0.3 \mu \mathrm{m}$ ) barriers are much smaller than e-PTFE $(0.5-30 \mu \mathrm{m})$, d-PTFE barriers provide a superior resistance to bacterial penetration. A pore size of less than $0.3 \mu \mathrm{m}$ (Bartee \& Carr, 1995) is impervious to bacteria, as the average size of bacteria is approximately $0.5-5.0 \mu \mathrm{m}$. A larger pore size allows for easy bacterial contamination once a membrane is exposed to the oral cavity. The presence of bacterial contamination compromises the integrity of the membrane and limits the regeneration process. Once compromised, the soft tissue begins to infiltrate the membrane, resulting in a much more difficult removal of the membrane and less favorable outcomes (Rakhmatia et al., 2013).

TABLE 2 Risk of bias assessment for the included RCT

\begin{tabular}{|c|c|c|c|}
\hline $\begin{array}{l}\text { Criteria, Higgins, and Green } \\
\text { (2011) }\end{array}$ & $\begin{array}{l}\text { Gher } \\
\text { et al. } \\
(1994)\end{array}$ & $\begin{array}{l}\text { Park } \\
\text { et al. } \\
(2008)\end{array}$ & $\begin{array}{l}\text { Fu et al. } \\
(2014)\end{array}$ \\
\hline Sequence generation & Yes & Yes & Yes \\
\hline Randomization methods & $\mathrm{RCT}$ & $\mathrm{RCT}$ & $\mathrm{RCT}$ \\
\hline $\begin{array}{l}\text { Allocation concealment } \\
\text { method }\end{array}$ & $?$ & Yes & Yes \\
\hline Examiner masked & $?$ & Yes & No \\
\hline $\begin{array}{l}\text { All patients accounted for at } \\
\text { end of study }\end{array}$ & Yes & Yes & Yes \\
\hline $\begin{array}{l}\text { Incomplete outcome data } \\
\text { adequately addressed }\end{array}$ & Yes & Yes & Yes \\
\hline $\begin{array}{l}\text { Free of suggestion of } \\
\text { selective outcome reporting }\end{array}$ & Yes & Yes & Yes \\
\hline $\begin{array}{l}\text { Estimated potential risk of } \\
\text { bias }\end{array}$ & High & Low & Moderate \\
\hline
\end{tabular}

?, Not reported.
Regarding GBR at peri-implant dehiscence sites, our study analyzed five articles (Annibali et al., 2012; Fu et al., 2014; Nowzari \& Slots, 1995; Park et al., 2008; Tawil et al., 2001) that included 87 surgical sites, and found $27 \%$ more defect reduction at sites without membrane exposure compared to those with exposures. This difference, however, is even more critical when considering absorbable (Fu et al., 2014; Park et al., 2008; Tawil et al., 2001) vs. nonresorbable membranes. As absorbable membranes are primarily metabolized through enzymatic degradation, once they become exposed, these membranes have a greater susceptibility to infection and a faster degradation rate. Membrane exposures compromise space maintenance and cell exclusion properties, leading to detrimental effects that are readily seen in subsequent outcomes. It has been reported that bacterial invasion of the exposed absorbable membrane could occur as early as 3 weeks (Simion et al., 1997). During the first week of exposure, the outer surface of the barrier is colonized by bacteria, and by week three to four, the bacteria have invaded the entire thickness of the membrane. Bacterial invasion results in membrane resorption and creation of irregular voids in the barrier, which degrade the functional integrity of the barrier. Even with the long-lasting cross-linked collagen barriers, Tal, Kozlovsky, Artzi, Nemcovsky, and Moses (2008) found that bone regeneration outcomes were compromised once membranes were exposed.

Interestingly, one study (Park et al., 2008) found a significant difference in peri-implant dehiscence defect reduction between sites with and without barrier exposure in a collagen membrane group but not in an ADM group. Four of six sites with ADM exposure were epithelialized after a 1-month period. The authors attributed this favorable outcome to the polarized matrix nature of ADM, which consisted of a basal lamina for epithelial cell migration and an underlying porous dermal matrix for angiogenic cell ingrowth (Cummings, Kaldahl, \& Allen, 2005). However, although the difference was not statistically significant, it is worth noting that $25 \%$ less dehiscence defect reduction was still detected in the exposed ADM group than the nonexposed group. 
TABLE 3 Risk of bias assessment for the included case series using the Methodological Index for Non-Randomized Studies (MINORS)

\begin{tabular}{|c|c|c|c|c|c|}
\hline $\begin{array}{l}\text { Criteria, Slim et al. } \\
\text { (2003) }\end{array}$ & $\begin{array}{l}\text { Buser } \\
\text { et al. } \\
\text { (1990) }\end{array}$ & $\begin{array}{l}\text { Nowzari and } \\
\text { Slots (1995) }\end{array}$ & $\begin{array}{l}\text { Chiapasco } \\
\text { et al. (1999) }\end{array}$ & $\begin{array}{l}\text { Tawil et al. } \\
\text { (2001) }\end{array}$ & $\begin{array}{l}\text { Annibali } \\
\text { et al. (2012) }\end{array}$ \\
\hline $\begin{array}{l}\text { A clearly stated } \\
\text { aim }\end{array}$ & 2 & 2 & 2 & 2 & 2 \\
\hline $\begin{array}{l}\text { Inclusion of } \\
\text { consecutive } \\
\text { patients }\end{array}$ & 2 & 2 & 2 & 2 & 2 \\
\hline $\begin{array}{l}\text { Prospective } \\
\text { collection of data }\end{array}$ & 2 & 2 & 2 & 2 & 0 \\
\hline $\begin{array}{l}\text { Endpoints } \\
\text { appropriate to } \\
\text { the aim of the } \\
\text { study }\end{array}$ & 2 & 2 & 2 & 2 & 2 \\
\hline $\begin{array}{l}\text { Unbiased } \\
\text { assessment of } \\
\text { the study } \\
\text { endpoint }\end{array}$ & 2 & 2 & 2 & 2 & 2 \\
\hline $\begin{array}{l}\text { Follow-up period } \\
\text { appropriate to } \\
\text { the aim of the } \\
\text { study }\end{array}$ & 2 & 2 & 2 & 2 & 2 \\
\hline $\begin{array}{l}\text { Loss to follow-up } \\
\text { less than } 5 \%\end{array}$ & 2 & 2 & 2 & 2 & 2 \\
\hline $\begin{array}{l}\text { Prospective } \\
\text { calculation of the } \\
\text { study size }\end{array}$ & 0 & 0 & 0 & 0 & 0 \\
\hline $\begin{array}{l}\text { Estimated } \\
\text { potential risk of } \\
\text { bias }\end{array}$ & Moderate & Moderate & Moderate & Moderate & High \\
\hline
\end{tabular}

2, Adequate; 1, Inadequate; 0, Not reported.

Our study findings are consistent with several previously published human studies reporting on membrane exposure, which show early membrane removal and compromised bone regeneration (von Arx, Hardt, \& Wallkamm, 1996; Proussaefs \& Lozada, 2006). It has been demonstrated that the timely membrane removal within 4 weeks of membrane exposure could still potentially result in successful bone augmentation (Annibali et al., 2012; Proussaefs \& Lozada, 2006). Annibali et al. (2012) treated patients with systemic antibiotic therapy ( $1 \mathrm{~g}$ amoxicillin-clavulanate, twice a day for 6 days) when membrane exposure occurred. The membrane was subsequently removed after reentry surgery, and the flap was closed. The graft was allowed to heal for an additional period ranging from 3 to 8 months. The study outcome showed a comparable amount of defect reduction between sites with and without membrane exposure. Therefore, proper management of membrane exposure could minimize the negative outcomes.

Both peri-implant and edentulous sites exhibit a significant decrease in regeneration outcomes if membranes become exposed. Therefore, it can be concluded that healing with primary intention is a crucial factor for GBR procedures. Chao et al. (2015) reported the soft tissue quality, flap flexibility, as well as the types of barrier membranes used have an important impact on wound opening. Ideally, an adequate amount of keratinized tissue, a thick tissue biotype, a deep vestibular depth, and high flap flexibility with a use of absorbable membranes might minimize the incidence of wound opening. The authors also reported that the use of nonresorbable membranes potentially has higher risk of membrane exposure compared to absorbable ones due to a tendency to revert to their original shape after being molded. However, our search did not identify clinical data on d-PTFE or absorbable synthetic membranes; thus, the influence of exposure of these types of barriers after GBR procedures on clinical outcomes cannot be determined at this time.

Other than membrane exposure, another important factor to be considered is that of soft tissue dehiscence at surgical sites. Two included studies (Fu et al., 2014; Park et al., 2008) had a control group for grafting materials alone without utilizing a barrier membrane. Fu et al. (2014) reported a detrimental effect of wound dehiscence $(-2.46 \%$ of defect height reduction and $-15.48 \%$ of defect width reduction) on the surgical outcome. However, Park et al. (2008) did not find a significant difference in defect height reduction and bone thickness gain between the sites with and without wound dehiscence. The authors attributed this outcome to the use of the "sandwich bone augmentation" technique. Previous studies (Lorenzoni, Pertl, Keil, \& Wegscheider, 1998; Lorenzoni et al., 2002) have confirmed that an absence of soft tissue dehiscence caused significant gain in the quantity of bone augmentation. Therefore, it can be concluded that both wound dehiscence and membrane exposure are contributing factors 
to unfavorable grafting outcomes. Clinically, achieving tension-free primary closure and wound stability to minimize the chance of wound dehiscence and membrane exposure is important (Lim, Lin, Monje, Chan, \& Wang, 2017).

There are several limitations in the current study. First, only eight papers were included in the current study. Second, most analyses were presented with high heterogeneity due to the different study designs, follow-up periods, materials used, etc. Third, all the included studies were deemed to have moderate to high risk of bias. This could be explained by the difficulty of conducting prospective studies to analyze outcomes on membrane exposure as this type of adverse event is hard to predict. Fourth, patient-centered outcomes were not analyzed in the current review due to the limited data. Fifth, our study only identified data on e-PTFE and collagen membranes. Future studies investigating the impact of membrane exposure with other types of currently available barriers, that is d-PTFE, on clinical parameters are needed.

\section{5 | CONCLUSION}

Based on the findings of the current study, membrane exposure after GBR procedures has a significant detrimental influence on the amount of bone augmentation. For GBR at edentulous sites, sites without membrane exposure achieved $74 \%$ more horizontal bone gain than sites with exposure. For peri-implant dehiscence defects, the sites without membrane exposure had $27 \%$ more defect reduction than sites with exposure. However, these outcomes are associated with collagen membranes and e-PTFE barriers. Future studies investigating the impact of membrane exposure with other types of currently available barriers on clinical parameters are needed.

\section{ORCID}

Hom-Lay Wang (iD http://orcid.org/0000-0003-4238-1799

Guo-Hao Lin (iD http://orcid.org/0000-0003-1290-9994

\section{REFERENCES OF ARTICLES INCLUDED IN QUALITATIVE ANALYSIS}

Annibali, S., Bignozzi, I., Sammartino, G., La Monaca, G., \& Cristalli, M. P. (2012). Horizontal and vertical ridge augmentation in localized alveolar deficient sites: A retrospective case series. Implant Dentistry, 21, 175-185. https://doi.org/10.1097/id.0b013e31824ee3e9

Buser, D., Bragger, U., Lang, N. P., \& Nyman, S. (1990). Regeneration and enlargement of jaw bone using guided tissue regeneration. Clinical Oral Implants Research, 1,22-32. https://doi.org/10.1034/j.1600-0501.1990.010104.x

Chiapasco, M., Abati, S., Romeo, E., \& Vogel, G. (1999). Clinical outcome of autogenous bone blocks or guided bone regeneration with e-ptfe membranes for the reconstruction of narrow edentulous ridges. Clinical Oral Implants Research, 10, 278-288. https://doi. org/10.1034/j.1600-0501.1999.100404.x

Fu, J. H., Oh, T. J., Benavides, E., Rudek, I., \& Wang, H. L. (2014). A randomized clinical trial evaluating the efficacy of the sandwich bone augmentation technique in increasing buccal bone thickness during implant placement surgery: I. Clinical and radiographic parameters. Clinical Oral Implants Research, 25, 458-467. https://doi. org/10.1111/clr.12171

Gher, M. E., Quintero, G., Assad, D., Monaco, E., \& Richardson, A. C. (1994). Bone grafting and guided bone regeneration for immediate dental implants in humans. Journal of Periodontology, 65, 881-891. https://doi. org/10.1902/jop.1994.65.9.881

Nowzari, H., \& Slots, J. (1995). Microbiologic and clinical study of polytetrafluoroethylene membranes for guided bone regeneration around implants. The International Journal of Oral \& Maxillofacial Implants, 10 , 67-73

Park, S. H., Lee, K. W., Oh, T. J., Misch, C. E., Shotwell, J., \& Wang, H. L. (2008). Effect of absorbable membranes on sandwich bone augmentation. Clinical Oral Implants Research, 19, 32-41. https://doi. org/10.1111/j.1600-0501.2007.01408.x

Tawil, G., El-Ghoule, G., \& Mawla, M. (2001). Clinical evaluation of a bilayered collagen membrane (bio-gide) supported by autografts in the treatment of bone defects around implants. The International Journal of Oral \& Maxillofacial Implants, 16, 857-863.

\section{REFERENCES}

Allen, E. P., Gainza, C. S., Farthing, G. G., \& Newbold, D. A. (1985). Improved technique for localized ridge augmentation. A report of 21 cases. Journal of Periodontology, 56, 195-199. https://doi.org/10.1902/ jop.1985.56.4.195

Antoun, H., Sitbon, J. M., Martinez, H., \& Missika, P. (2001). A prospective randomized study comparing two techniques of bone augmentation: Onlay graft alone or associated with a membrane. Clinical Oral Implants Research, 12, 632-639. https://doi. org/10.1034/j.1600-0501.2001.120612.x

von Arx, T., \& Buser, D. (2006). Horizontal ridge augmentation using autogenous block grafts and the guided bone regeneration technique with collagen membranes: A clinical study with 42 patients. Clinical Oral Implants Research, 17, 359-366. https://doi. org/10.1111/j.1600-0501.2005.01234.x

von Arx, T., Hardt, N., \& Wallkamm, B. (1996). The time technique: A new method for localized alveolar ridge augmentation prior to placement of dental implants. The International Journal of Oral \& Maxillofacial Implants, 11, 387-394.

von Arx, T., \& Kurt, B. (1999). Implant placement and simultaneous ridge augmentation using autogenous bone and a micro titanium mesh: A prospective clinical study with $20 \mathrm{im}$ plants. Clinical Oral Implants Research, 10, 24-33. https://doi. org/10.1034/j.1600-0501.1999.100104.x

Assenza, B., Piattelli, M., Scarano, A., Lezzi, G., Petrone, G., \& Piattelli, A. (2001). Localized ridge augmentation using titanium micromesh. Journal of Oral Implantology, 27, 287-292. https://doi.org/10.1563/15481336(2001) 027<0287:LRAUTM>2.3.CO;2

Bahat, O., \& Fontanessi, R. V. (2001). Implant placement in threedimensional grafts in the anterior jaw. The International Journal of Periodontics \& Restorative Dentistry, 21, 357-365. https://doi. org/10.11607/prd.00.0423

Bartee, B. K., \& Carr, J. A. (1995). Evaluation of a high-density polytetrafluoroethylene (n-PTFE) membrane as a barrier material to facilitate guided bone regeneration in the rat mandible. Journal of Oral Implantology, 21, 88-95.

Buser, D., Dula, K., Hirt, H. P., \& Schenk, R. K. (1996). Lateral ridge augmentation using autografts and barrier membranes: A clinical study with 40 partially edentulous patients. Journal of Oral \& Maxillofacial Surgery, 54, 420-432; discussion 432-423. https://doi.org/10.1016/ s0278-2391(96)90113-5

Buser, D., Martin, W., \& Belser, U. C. (2004). Optimizing esthetics for implant restorations in the anterior maxilla: Anatomic and surgical considerations. The International Journal of Oral \& Maxillofacial Implants, 19(Suppl), 43-61. 
Chao, Y. C., Chang, P. C., Fu, J. H., Wang, H. L., \& Chan, H. L. (2015). Surgical site assessment for soft tissue management in ridge augmentation procedures. The International Journal of Periodontics \& Restorative Dentistry, 35, e75-e83. https://doi.org/10.11607/prd.2097

Chiapasco, M., Autelitano, L., Rabbiosi, D., \& Zaniboni, M. (2013). The role of pericranium grafts in the reduction of postoperative dehiscences and bone resorption after reconstruction of severely deficient edentulous ridges with autogenous onlay bone grafts. Clinical Oral Implants Research, 24, 679-687. https://doi.org/10.1111/j.1600-0501.2012.02485.x

Chiapasco, M., Romeo, E., \& Vogel, G. (2001). Vertical distraction osteogenesis of edentulous ridges for improvement of oral implant positioning: A clinical report of preliminary results. The International Journal of Oral \& Maxillofacial Implants, 16, 43-51.

Chin, M. (1999). Distraction osteogenesis for dental implants. Atlas of the Oral and Maxillofacial Surgery Clinics of North America, 7, 41-63.

Cordaro, L., Amade, D. S., \& Cordaro, M. (2002). Clinical results of alveolar ridge augmentation with mandibular block bone grafts in partially edentulous patients prior to implant placement. Clinical Oral Implants Research, 13, 103-111. https://doi.org/10.1034/j.1600-0501.2002.130113.x

Cordaro, L., Torsello, F., Morcavallo, S., \& di Torresanto, V. M. (2011). Effect of bovine bone and collagen membranes on healing of mandibular bone blocks: A prospective randomized controlled study. Clinical Oral Implants Research, 22, 1145-1150. https://doi. org/10.1111/j.1600-0501.2010.02093.x

Cummings, L. C., Kaldahl, W. B., \& Allen, E. P. (2005). Histologic evaluation of autogenous connective tissue and acellular dermal matrix grafts in humans. Journal of Periodontology, 76, 178-186. https://doi. org/10.1902/jop.2005.76.2.178

Friedmann, A., Strietzel, F. P., Maretzki, B., Pitaru, S., \& Bernimoulin, J. P. (2002). Histological assessment of augmented jaw bone utilizing a new collagen barrier membrane compared to a standard barrier membrane to protect a granular bone substitute material. Clinical Oral Implants Research, 13, 587-594. https://doi. org/10.1034/j.1600-0501.2002.130603.x

Greenstein, G., \& Carpentieri, J. R. (2015). Utilization of d-ptfe barriers for post-extraction bone regeneration in preparation for dental implants. Compendium of Continuing Education, 36, 465-473.

Her, S., Kang, T., \& Fien, M. J. (2012). Titanium mesh as an alternative to a membrane for ridge augmentation. Journal of Oral \& Maxillofacial Surgery, 70, 803-810. https://doi.org/10.1016/j.joms.2011.11.017

Higgins, J. P., \& Green, S.. (2011) Cochrane Handbook for Systematic Reviews of Interventions Version 5.1.0 [updated March 2011]. The Cochrane Collaboration, 2011. Retrieved from www.cochrane-handbook.org.

Hoffmann, O., Bartee, B. K., Beaumont, C., Kasaj, A., Deli, G., \& Zafiropoulos, G. G. (2008). Alveolar bone preservation in extraction sockets using non-resorbable dptfe membranes: A retrospective nonrandomized study. Journal of Periodontology, 79, 1355-1369. https:// doi.org/10.1902/jop.2008.070502

Kfir, E., Kfir, V., \& Kaluski, E. (2007). Immediate bone augmentation after infected tooth extraction using titanium membranes. Journal of Oral Implantology, 33, 133-138. https://doi.org/10.1563/1548-1336(2007) 33[133:IBAAIT]2.0.CO;2

Lee, J. Y., Kim, Y. K., Yi, Y. J., \& Choi, J. H. (2013). Clinical evaluation of ridge augmentation using autogenous tooth bone graft material: Case series study. Journal of the Korean Association of Oral and Maxillofacial Surgeons, 39, 156-160. https://doi.org/10.5125/jkaoms.2013.39.4.156

Liberati, A., Altman, D. G., Tetzlaff, J., Mulrow, C., Gotzsche, P. C., loannidis, J. P., ... Moher, D. (2009). The prisma statement for reporting systematic reviews and meta-analyses of studies that evaluate health care interventions: Explanation and elaboration. Journal of Clinical Epidemiology, 62, e1-e34. https://doi.org/10.1016/j.jclinepi.2009.06.006

Lim, G., Lin, G. H., Monje, A., Chan, H. L., \& Wang, H. L. (2017). Wound healing complications following guided bone regeneration for ridge augmentation: A systematic review and meta-analysis. The International Journal of Oral \& Maxillofacial Implants, 33, 41-50.
Lindfors, L. T., Tervonen, E. A., Sandor, G. K., \& Ylikontiola, L. P. (2010). Guided bone regeneration using a titanium-reinforced eptfe membrane and particulate autogenous bone: The effect of smoking and membrane exposure. Oral Surgery, Oral Medicine, Oral Pathology, Oral Radiology \& Endodontology, 109, 825-830. https://doi.org/10.1016/j. tripleo.2009.12.035

Lizio, G., Corinaldesi, G., \& Marchetti, C. (2014). Alveolar ridge reconstruction with titanium mesh: A three-dimensional evaluation of factors affecting bone augmentation. The International Journal of Oral \& Maxillofacial Implants, 29, 1354-1363. https://doi.org/10.11607/ jomi.3417

Lorenzoni, M., Pertl, C., Keil, C., \& Wegscheider, W. A. (1998). Treatment of peri-implant defects with guided bone regeneration: A comparative clinical study with various membranes and bone grafts. The International Journal of Oral \& Maxillofacial Implants, 13, 639-646.

Lorenzoni, M., Pertl, C., Polansky, R. A., Jakse, N., \& Wegscheider, W. A. (2002). Evaluation of implants placed with barrier membranes. A retrospective follow-up study up to five years. Clinical Oral Implants Research, 13, 274-280. https://doi.org/10.1034/ j.1600-0501.2002.130306.x

Louis, P. J., Gutta, R., Said-Al-Naief, N., \& Bartolucci, A. A. (2008). Reconstruction of the maxilla and mandible with particulate bone graft and titanium mesh for implant placement. Journal of Oral \& Maxillofacial Surgery, 66, 235-245. https://doi.org/10.1016/j.joms.2007.08.022

Lyford, R. H., Mills, M. P., Knapp, C. I., Scheyer, E. T., \& Mellonig, J. T. (2003). Clinical evaluation of freeze-dried block allografts for alveolar ridge augmentation: A case series. The International Journal of Periodontics \& Restorative Dentistry, 23, 417-425. https://doi.org/10.11607/prd.00.0543

Machtei, E. E. (2001). The effect of membrane exposure on the outcome of regenerative procedures in humans: A meta-analysis. Journal of Periodontology, 72, 512-516. https://doi.org/10.1902/ jop.2001.72.4.512

Mattout, P., \& Mattout, C. (2000). Conditions for success in guided bone regeneration: Retrospective study on 376 implant sites. Journal of Periodontology, 71, 1904-1909. https://doi.org/10.1902/ jop.2000.71.12.1904

Mellonig, J. T., \& Nevins, M. (1995). Guided bone regeneration of bone defects associated with implants: An evidence-based outcome assessment. The International Journal of Periodontics \& Restorative Dentistry, 15, 168-185. https://doi.org/10.11607/prd.00.0122

Meloni, S. M., Jovanovic, S. A., Urban, I., Canullo, L., Pisano, M., \& Tallarico, M. (2017). Horizontal ridge augmentation using gbr with a native collagen membrane and 1:1 ratio of particulated xenograft and autologous bone: A 1-year prospective clinical study. Clinical Implant Dentistry and Related Research, 19, 38-45. https://doi.org/10.1111/cid.12429

Merli, M., Moscatelli, M., Mariotti, G., Pagliaro, U., Raffaelli, E., \& Nieri, M. (2015). Comparing membranes and bone substitutes in a onestage procedure for horizontal bone augmentation. A double-blind randomised controlled trial. European Journal of Oral Implantology, 8, 271-281.

Misch, C. M. (1997). Comparison of intraoral donor sites for onlay grafting prior to implant placement. The International Journal of Oral \& Maxillofacial Implants, 12, 767-776.

Miyamoto, I., Funaki, K., Yamauchi, K., Kodama, T., \& Takahashi, T. (2012). Alveolar ridge reconstruction with titanium mesh and autogenous particulate bone graft: Computed tomography-based evaluations of augmented bone quality and quantity. Clinical Implant Dentistry and Related Research, 14, 304-311. https://doi. org/10.1111/j.1708-8208.2009.00257.x

Park, S. H., Brooks, S. L., Oh, T. J., \& Wang, H. L. (2009). Effect of ridge morphology on guided bone regeneration outcome: Conventional tomographic study. Journal of Periodontology, 80, 1231-1236. https://doi. org/10.1902/jop.2009.090090

Parodi, R., Carusi, G., Santarelli, G., \& Nanni, F. (1998). Implant placement in large edentulous ridges expanded by gbr using a bioresorbable 
collagen membrane. The International Journal of Periodontics \& Restorative Dentistry, 18, 266-275. https://doi.org/10.11607/ prd.00.0269

Proussaefs, P., \& Lozada, J. (2003). The use of resorbable collagen membrane in conjunction with autogenous bone graft and inorganic bovine mineral for buccal/labial alveolar ridge augmentation: A pilot study. Journal of Prosthetic Dentistry, 90, 530-538. https://doi.org/10.1016/ S0022391303005213

Proussaefs, P., \& Lozada, J. (2006). Use of titanium mesh for staged localized alveolar ridge augmentation: Clinical and histologic-histomorphometric evaluation. Journal of Oral Implantology, 32, 237-247. https://doi. org/10.1563/1548-1336(2006) 32[237:UOTMFS]2.0.CO;2

Proussaefs, P., Lozada, J., Kleinman, A., Rohrer, M. D., \& McMillan, P. J. (2003). The use of titanium mesh in conjunction with autogenous bone graft and inorganic bovine bone mineral (Bio-Oss) for localized alveolar ridge augmentation: A human study. The International Journal of Periodontics \& Restorative Dentistry, 23, 185-195. https://doi. org/10.11607/prd.00.0515

Rakhmatia, Y. D., Ayukawa, Y., Furuhashi, A., \& Koyano, K. (2013). Current barrier membranes: Titanium mesh and other membranes for guided bone regeneration in dental applications. Journal of Prosthodontic Research, 57, 3-14. https://doi.org/10.1016/j.jpor.2012.12.001

Seibert, J. S. (1983). Reconstruction of deformed, partially edentulous ridges, using full thickness onlay grafts. Part I. Technique and wound healing. Compendium of Continuing Education, 4, 437-453.

Sethi, A., \& Kaus, T. (2001). Ridge augmentation using mandibular block bone grafts: Preliminary results of an ongoing prospective study. The International Journal of Oral \& Maxillofacial Implants, 16, 378-388.

Shea, B. J., Grimshaw, J. M., Wells, G. A., Boers, M., Andersson, N., Hamel, C., ... Bouter, L. M. (2007). Development of amstar: A measurement tool to assess the methodological quality of systematic reviews. BMC Medical Research Methodology, 7, 10. https://doi.org/10.1186/1471-2288-7-10

Simion, M., Baldoni, M., \& Zaffe, D. (1992). Jawbone enlargement using immediate implant placement associated with a split-crest technique and guided tissue regeneration. The International Journal of Periodontics \& Restorative Dentistry, 12, 462-473. https://doi.org/10.11607/ prd.00.0036

Simion, M., Maglione, M., lamoni, F., Scarano, A., Piattelli, A., \& Salvato, A. (1997). Bacterial penetration through resolut resorbable membrane in vitro. An histological and scanning electron microscopic study. Clinical Oral Implants Research, 8, 23-31. https://doi. org/10.1111/j.1600-0501.1997.tb00004.x

Slim, K., Nini, E., Forestier, D., Kwiatkowski, F., Panis, Y., \& Chipponi, J. (2003). Methodological index for non-randomized studies (minors): Development and validation of a new instrument. Australian and New Zealand Journal of Surgery, 73, 712-716. https://doi. org/10.1046/j.1445-2197.2003.02748.x

Spray, J. R., Black, C. G., Morris, H. F., \& Ochi, S. (2000). The influence of bone thickness on facial marginal bone response: Stage 1 placement through stage 2 uncovering. Annals of Periodontology, 5, 119-128. https://doi.org/10.1902/annals.2000.5.1.119

Tal, H., Kozlovsky, A., Artzi, Z., Nemcovsky, C. E., \& Moses, O. (2008). Longterm bio-degradation of cross-linked and non-cross-linked collagen barriers in human guided bone regeneration. Clinical Oral Implants Research, 19, 295-302. https://doi.org/10.1111/j.1600-0501.2007.01424.x

Tarnow, D. P., Cho, S. C., \& Wallace, S. S. (2000). The effect of interimplant distance on the height of inter-implant bone crest. Journal of Periodontology, 71, 546-549. https://doi.org/10.1902/ jop.2000.71.4.546
Torres, J., Tamimi, F., Alkhraisat, M. H., Manchon, A., Linares, R., PradosFrutos, J. C., ... Lopez Cabarcos, E. (2010). Platelet-rich plasma may prevent titanium-mesh exposure in alveolar ridge augmentation with anorganic bovine bone. Journal of Clinical Periodontology, 37, 943-951. https://doi.org/10.1111/j.1600-051X.2010.01615.x

Uehara, S., Kurita, H., Shimane, T., Sakai, H., Kamata, T., Teramoto, Y., \& Yamada, S. (2015). Predictability of staged localized alveolar ridge augmentation using a micro titanium mesh. Oral \& Maxillofacial Surgery, 19, 411-416. https://doi.org/10.1007/s10006-015-0513-6

Urban, I. A., Nagursky, H., \& Lozada, J. L. (2011). Horizontal ridge augmentation with a resorbable membrane and particulated autogenous bone with or without anorganic bovine bone-derived mineral: A prospective case series in 22 patients. The International Journal of Oral \& Maxillofacial Implants, 26, 404-414.

Urban, I. A., Nagursky, H., Lozada, J. L., \& Nagy, K. (2013). Horizontal ridge augmentation with a collagen membrane and a combination of particulated autogenous bone and anorganic bovine bone-derived mineral: A prospective case series in 25 patients. The International Journal of Periodontics \& Restorative Dentistry, 33, 299-307. https://doi. org/10.11607/prd.1407

Waasdorp, J., \& Feldman, S. (2013). Bone regeneration around immediate implants utilizing a dense polytetrafluoroethylene membrane without primary closure: A report of 3 cases. Journal of Oral Implantology, 39, 355-361. https://doi.org/10.1563/AAID-JOI-D-10-00128

Wang, H. L., \& Al-Shammari, K. (2002). HVC ridge deficiency classification: A therapeutically oriented classification. The International Journal of Periodontics \& Restorative Dentistry, 22, 335-343. https://doi. org/10.11607/prd.00.0481

Wang, H. L., \& Boyapati, L. (2006). "PASS" principles for predictable bone regeneration. Implant Dentistry, 15, 8-17. https://doi.org/10.1097/01. id.0000204762.39826.0f

Wang, H. L., Misch, C., \& Neiva, R. F. (2004). "Sandwich" bone augmentation technique: Rationale and report of pilot cases. The International Journal of Periodontics \& Restorative Dentistry, 24, 232-245.

Wessing, B., Emmerich, M., \& Bozkurt, A. (2016). Horizontal ridge augmentation with a novel resorbable collagen membrane: A retrospective analysis of 36 consecutive patients. The International Journal of Periodontics \& Restorative Dentistry, 36, 179-187. https://doi. org/10.11607/prd.2065

Zitzmann, N. U., Naef, R., \& Scharer, P. (1997). Resorbable versus nonresorbable membranes in combination with bio-oss for guided bone regeneration. The International Journal of Oral \& Maxillofacial Implants, 12, 844-852.

\section{SUPPORTING INFORMATION}

Additional Supporting Information may be found online in the supporting information tab for this article.

How to cite this article: Garcia J, Dodge A, Luepke P, Wang $\mathrm{H}-\mathrm{L}$, Kapila Y, Lin G-H. Effect of membrane exposure on guided bone regeneration: A systematic review and metaanalysis. Clin Oral Impl Res. 2018;29:328-338. https://doi. org/10.1111/clr.13121 